\title{
SMART EXERCISE APPLICATION TO IMPROVE LEG FUNCTION AND SHORT-TERM MEMORY THROUGH GAME- LIKE LUNGE EXERCISES: DEVELOPMENT AND EVALUATION
}

\author{
Byungjoo Noh $^{1,4}$, Eric Vasey ${ }^{2}$, Kevin Phillips ${ }^{1}$, Myounghoon Jeon ${ }^{2,3,5}$, and Tejin Yoon ${ }^{1,6}$ \\ ${ }^{1}$ Department of Kinesiology and Integrative Physiology, Michigan Technological University, \\ Houghton, Michigan, USA \\ ${ }^{2}$ Department of Computer Science, Michigan Technological University, Houghton, USA \\ ${ }^{3}$ Department of Cognitive and Learning Sciences, Michigan Technological University, \\ Houghton, USA \\ ${ }^{4}$ Department of Health Care and Science, Dong-A University, Busan, Republic of Korea \\ ${ }^{5}$ Department of Industrial and Systems Engineering, Virginia Tech, Blacksburg, USA \\ ${ }^{6}$ Department of Physical Education, Kangwon National University, Chuncheon, \\ Republic of Korea
}

Original scientific paper

https://doi.org/10.26582/k.51.1.10

\begin{abstract}
:
The purpose of this study was to evaluate the functionality, accuracy, and usability of a novel smart exercise application (SEA). The functionality such as counting lunges, providing task-related auditory feedback, and testing short-term memory was examined while thirteen young adults (six men, age $25.4 \pm 8.3$ years) performed the lunge exercise with the SEA. The accuracy of logged motion data including angles and accelerations were also tested. Another twenty-five participants (11 men, age $23.2 \pm 5.7$ years) evaluated the usability of the SEA interest, motivation, convenience, and strength/cognitive benefit via a questionnaire. The SEA assessed the lunge motion correctly, provided auditory feedback, and tested users' short-term memory as required. High correlations $(\mathrm{r}=0.90$ to 0.99$)$ with low RMSE $\left(4.85^{\circ}\right.$ for direction angle, 0.13 to $0.22 \mathrm{~m} /$ $\mathrm{s}^{2}$ for acceleration) were observed between the sensor output and the reference output. Bland-Altman plot also showed a low discrepancy between each of the two measures. Most participants positively answered all questions about interest (60\%), motivation ( $40 \%$ ), convenience (80\%), strength benefits (92\%), and cognitive benefits $(88 \%)$ of the SEA. The SEA demonstrated accurate kinematic assessment of accelerations and directions, assessed the lunge motion correctly, and created the appropriate auditory feedback on the shortterm memory task. The high rate of positive responses suggested the potential of the application in future use.
\end{abstract}

Key words: auditory feedback, smart phone, home-based exercise, inertial measurement unit

\section{Introduction}

The lunge is a popular and common movement for training and rehabilitation of the lower limbs (Heijne, et al., 2004; Jönhagen, Halvorsen, \& Benoit, 2009). Despite the potential of a lunge exercise for home-based exercise, repeating a simple motion can get boring and make people sore. To overcome this limitation and promote self-guided lunge exercises, several Android and iOS applications such as "30 day lunge challenge (Creative Apps, Inc)", "How to do a proper lunge (Abi apps)", "Squats and lunges (Maplevine)", "Great legs (Fitness22, LTD)", and "Fast butt and legs (Olson applications, LTD)" have been recently introduced to the market. These applications provide tutorial pictures/audios/videos, count the number of lunge repetition, and allow monthly goals to be set to help users train by themselves. However, it is doubtful whether most applications are good enough to overcome the limitations mentioned above and draw users' interests given the lower number of downloads (100-50,000). These applications might be enhanced by adding interactive and game-like functions using task-related multimodal feedback, which has been known to enhance performance significantly during motion tracking exercises (Rosati, Oscari, Spagnol, Avan- 
zini, \& Masiero, 2012; Seizova-Cajic \& Azzi, 2010).

In addition to boredom issues, people without the proper knowledge may perform incorrect lunge motions, which could increase the incidence of injuries (Farrokhi, et al., 2008). It is, therefore, essential to ensure that people perform their exercise correctly based on an objective assessment. Generally, a biomechanical tool such as the Vicon motion analysis system (Oxford Metrics, UK) is a standard way to assess human movement, but it is very expensive, and requires knowledge, time and effort to get objective data. Recently, inertial measurement units (IMU) have been getting more popular because they are cost-effective, small, and easy to setup (Picerno, 2017). A number of studies have validated various human exercises including the lunge motion with IMU sensors. For example, Fitzgerald et al (2007) used ten IMUs to identify the difference between injured and non-injured athletes during the lunge. Other groups also tested the lunge motion using IMU(s) and showed good accuracy compared to their laboratory-based reference system (Leardini, et al., 2014; Tang, et al., 2015). IMUs were also used combined with a vision depth sensor and increased the rate of classification of the lunge motion overall up to $100 \%$ (Chen, Jafari, \& Kehtarnavaz, 2016; Gowing et al., 2014). In addition to sensor accuracy, one to five IMUs were used to discriminate between correct and incorrect performance of the lunges (O'Reilly, Whelan, Ward, Delahunt, \& Caulfield, 2017; Whelan, O'Reilly, Ward, Delahunt, \& Caulfield, 2016). All the above solutions can provide relatively accurate motion data. However, these PC-based systems are not portable and are still expensive.

Recently, O'Reilly et al. (O'Reilly, Duffin, Ward, \& Caulfield, 2017) developed a tablet application which successfully automated the process of creating an individualized exercise biofeedback system. This system seems to be an excellent solution for home-based exercise because it can be carried and still track and provide motion data. However, it is still expensive $(>\$ 500)$ and lacks real-time task-related feedback on motion. More functional but still cost-effective solutions are needed. Our long-term goal is to develop an appbased exercise application with a cheap wearable sensor $(<\$ 100)$ that still provides motion data therefore people can exercise by themselves whenever they want. In addition to physical training promotion, another main goal of our application is to improve cognitive function, particularly short-term memory. Short-term memory is a type of memory used to retain information for a short time (15-30 seconds) (Atkinson \& Shiffrin, 1971). Previous studies revealed that a square stepping exercise, where the users were asked to memorize the number of blocks and step on each block in order, could improve cognitive functions (Kitazawa, et al., 2015;
Teixeira, et al., 2013). To accomplish the abovementioned goals, it is necessary to have a userfriendly and well-functioning application. Thus, the primary purpose of this study was to evaluate the functionality of a novel smart exercise application (SEA) while young adults performed a lunge exercise. The second purpose was to evaluate usability of the SEA.

\section{Methods}

\section{Overview of the system}

A Bluetooth sensor (Mbientlab, Portola, San Francisco, CA) including a tri-axial accelerometer and a gyroscope was used to collect motion data on the Android platform. The application was developed in Android Studio (Ver. 2.3, Google) using the Java programming language. The application was set to track the lunge movements at a sampling frequency of $100 \mathrm{~Hz}$. In addition to the data collection, PureData (https://puredata.info/) patch was used to create auditory feedback about users' motion (Institute of Electronic Music and Acoustics, 2016). Real time task-related auditory feedback is known to be more effective for learning/ performing motion (Rosati, et al., 2012). We call this function the "lunge piano" because users can play sounds by pressing virtual piano keys while performing a clock lunge (i.e., users rotate their body to face a direction to select a key of the virtual piano), and play a sound by performing a correct lunge motion (see Supplementary video and Figure 1). We also added a game-like function, called "lunge memory" to motivate users and improve memory function as well. In this function users were given a sequence of notes with four sounds (e.g., "C-E-G-E" with graphical and text displays) and they must play each sound by changing their body direction and performing the lunges accurately in order.

\section{Participants}

Thirteen healthy young adults (six men, aged $25.4 \pm 8.3$ years, body weight $68.8 \pm 11.0 \mathrm{~kg}$, body height $169.3 \pm 8.5 \mathrm{~cm}$ ) volunteered to participate in the validation test and 25 young adults (11 men, aged $23.2 \pm 5.7$ years) participated in the usability test. After reading all details of the study, participants gave their informed consent. This study was approved by the University Institutional Review Board.

\section{Experimental setup and data acquisition}

To validate the motion data, research quality motion analysis tools were used as a reference. A wireless accelerometer (ACC; Trigno wireless, Delsys, Boston, MA) was attached next to the sensor on a custom-made platform using cardboard 
A

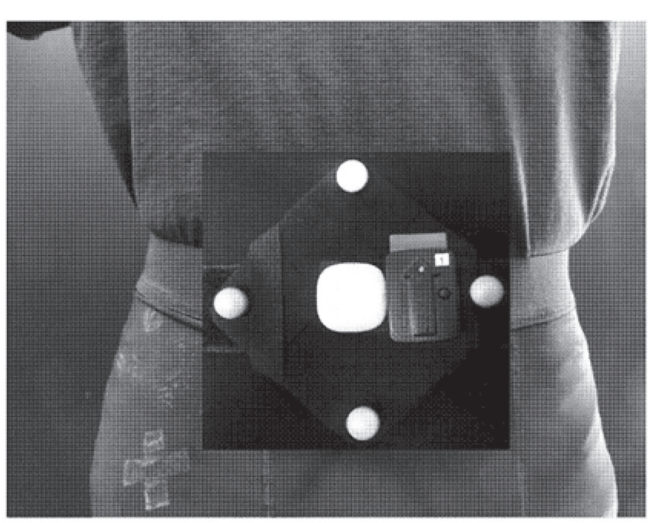

C

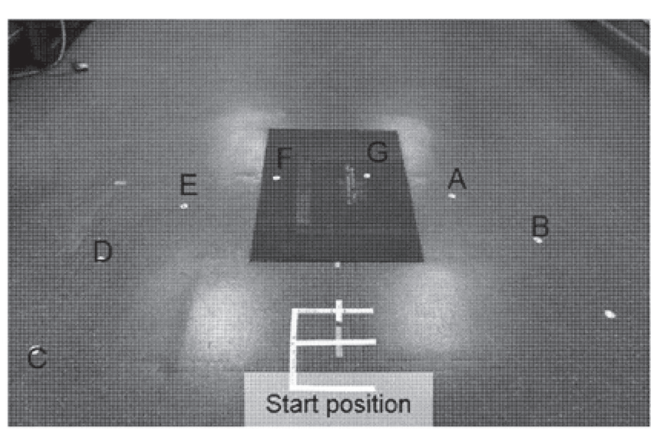

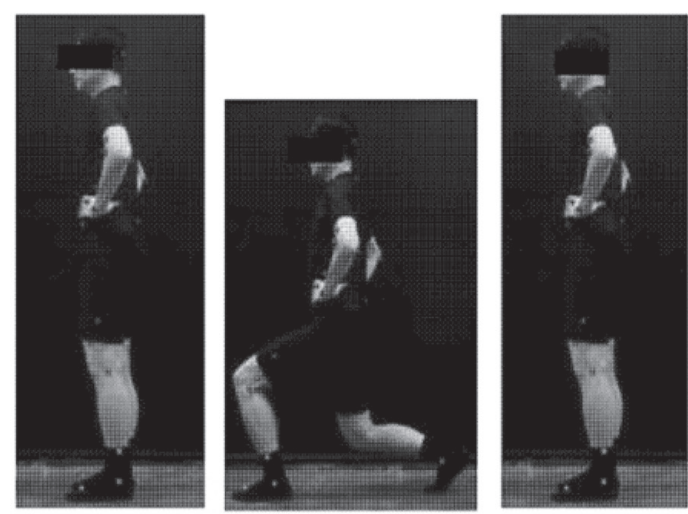

D

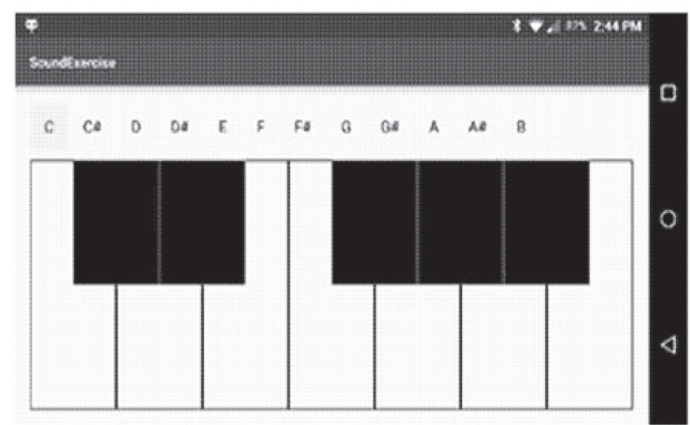

Figure 1. (A) IMU (center), 4 reflector markers (white, round), and accelerometer (black). (B) Lunge motion of the participant. (C) Guidelines on the floor with the name of sounds. (D) Screen shot of SEA.

and fixed on the abdomen of each participant (size: $90 \times 90 \mathrm{~mm}$ ) using a Velcro belt and double-sided adhesive tape before the experimental trial. Three directional accelerations were sampled at $150 \mathrm{~Hz}$ using the EMGworks program (Delsys, Boston, MA). Four reflective markers were also attached on the four corners of the platform surrounding the sensor. The positions of markers were sampled at $200 \mathrm{~Hz}$ using a three-dimensional motion capture (Mocap) system with eight cameras (OptiTrack Prime 13; Natural Point, Corvallis, OR).

\section{Procedure}

Participants were instructed to take a step forward, placing the right foot to a designated direction on the ground, flexing the knee until at a $90^{\circ}$ angle, then subsequently extending the right knee back into their initial standing position (Alkjær, Henriksen, Dyhre-Poulsen, \& Simonsen, 2009; Henriksen, Alkjær, Simonsen, \& Bliddal, 2009) (Figure 1B). To guide the landing position of the right foot, seven directions were marked on the ground. Each direction was separated by $25^{\circ}$ on the ground (Figure 1C). Each lunge motion was performed within 3-second duration, and the timing of motion was guided by a metronome. After a 5-minute practice, participants completed three sets of a barefoot forward lunge trial consisting of four lunges, in four different directions (12 lunges in total).

\section{Data analysis}

The best performance of the three sets was selected by two experimenters and all data during the same trials from different systems were analyzed respectively. The acceleration and angular velocity data from the sensor were passed through a Kalman filter (Kalman, 1960) to remove motion artifacts and electrical noise using the Kalman filter library (Sourceforge, 2016). After filtering, the relative angular displacement of the sensor from the initial position on the axial plane was used as an angular displacement. The calculated direction angle was then used to determine the note; for example, $0-25^{\circ}$ zone selects the note $\mathrm{C}$. The magnitude and duration of the sum of accelerations in two directions ( $\mathrm{Y}$ and Z: up-down and forward-backward, respectively) were calculated to determine if a lunge had been performed or not. The sound selected based on the direction angle was produced as feedback when a certain magnitude level was maintained for a while the lunge motion. For participants' different styles of the lunge motion, various combinations of the magnitude and duration were tested throughout the development process. Based on these preliminary results, the combination of $>12 \mathrm{~m} / \mathrm{s}^{2}$ of acceleration and $>0.1 \mathrm{~s}$ of duration condition was used in this study (i.e., when the acceleration of $12 \mathrm{~m} / \mathrm{s}^{2}$ or greater acceleration is maintained for at least $0.1 \mathrm{~s}$ ).

The acceleration signals from the ACC were lowpass Butterworth filtered with a cutoff frequency 
of $10 \mathrm{~Hz}$ using the EMGworks analysis program (Delsys, Inc., Boston, MA). The position data of each marker from the Mocap system were low-pass Butterworth filtered with a cutoff frequency of 10 $\mathrm{Hz}$. The direction angle of the platform on the axial plane was calculated using the cross-product of two vectors formed by three different markers.

The time of peak acceleration and direction angle during a standing jump was used to synchronize all systems (Acceleration: SEA vs. ACC; Direction angle: SEA vs. Mocap) prior to the start of each trial. All data were normalized to the lunge movement cycle using the interpolation function in MATLAB (MathWorks, Natick, MA). Signals from IMU sensor were converted to acceleration $\left(\mathrm{m} / \mathrm{s}^{2}\right)$ and angle (degrees) to compare with those from ACC and Mocap, respectively. Root-meansquare error (RMSE) was used to evaluate differences between the values from SEA and the values from the reference system.

\section{Usability of the SEA}

After a $\sim 10$-minute demonstration of the application, participants tested functions by themselves for 5-10 min and completed an anonymous modified survey which was validated by others (Brooke, 1996). It included a total of 11 questions about exercise frequency, experience with exercise applications, usability, interest, motivation, convenience, and strength/cognitive benefits of the SEA. Participants were asked to answer the questions along a 5-level Likert scale (strongly agree, agree, neutral, disagree, and strongly disagree).

\section{Statistical analysis}

Results are expressed as mean $\pm \mathrm{SD}$. Agreement between the 3-axes accelerations by the IMU and ACC and direction angle by the IMU and the Mocap system were evaluated by Pearson's correlation coefficient ( $r$-value). A Bland-Altman plot was also used to evaluate the discrepancy between measurements obtained from the two different systems. Mean bias and 95\% limits of agreement (LOA) were calculated for each of the acceleration and direction angles. The analysis was conducted using IBM SPSS Statistics 22. Values of $\mathrm{p}<.05$ were considered as statistically significant results.

\section{Results}

\section{Angle}

Measurements of the direction angles by the application and the Mocap demonstrated a mean RMSE of $4.85 \pm 1.34^{\circ}$. Pearson's correlation coefficient was $0.99 \pm 0.00(\mathrm{p}<.001)$ comparing the two measurements during forward lunges for all participants. Single participant data of direction angle is illustrated in Figure 2A. A Bland-Altman plot of the difference between the IMU and Mocap measure-

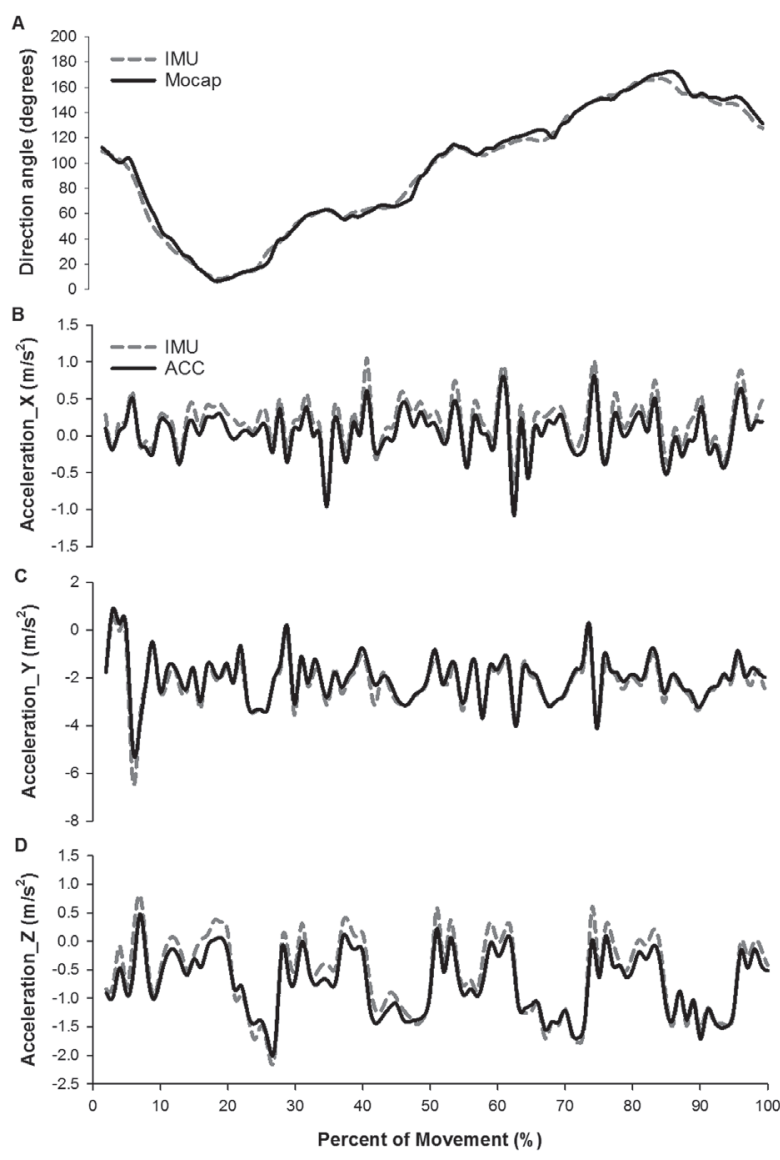

Figure 2. (A) Single participant output of direction angle comparison to reference system (Mocap). (B-D) Single participant output of 3-axes accelerations of the IMU comparison to reference system (ACC).

ments demonstrated a low discrepancy. The mean bias for direction angle was $0.61 \pm 3.34^{\circ}$ with $95 \%$ of LOA (Figure 3A).

\section{Accelerations}

Measurements of the 3-axes accelerations by the IMU and ACC resulted in a mean RMSE of $0.13 \pm 0.04 \mathrm{~m} / \mathrm{s}^{2}, 0.21 \pm 0.06 \mathrm{~m} / \mathrm{s}^{2}$, and $0.22 \pm 0.03 \mathrm{~m} /$ $\mathrm{s}^{2}$, respectively. Single participant data of acceleration is shown in Figure 2B-D. For x, y, and z-axes accelerations, the Pearson's correlation coefficients between two systems were $.90 \pm .08, .93 \pm .05$, and $.93 \pm .04$ ( $\mathrm{p}<.001$, respectively). The Bland-Altman plot also showed a low discrepancy between the two systems. The mean bias across all axes was $-0.01 \pm 0.01 \mathrm{~m} / \mathrm{s}^{2}$ with $95 \%$ of LOA (Figure 3B-D).

\section{Functionality}

All participants understood how to create sounds after 5 min of practice and our application created sounds $100 \%$ (total 156 lunge motions: 4 sounds $\times 3$ times $\times 13$ participants) accurately. The sequences of note and all data from accelerometer and gyroscope were logged correctly and could be delivered to a designated email. 

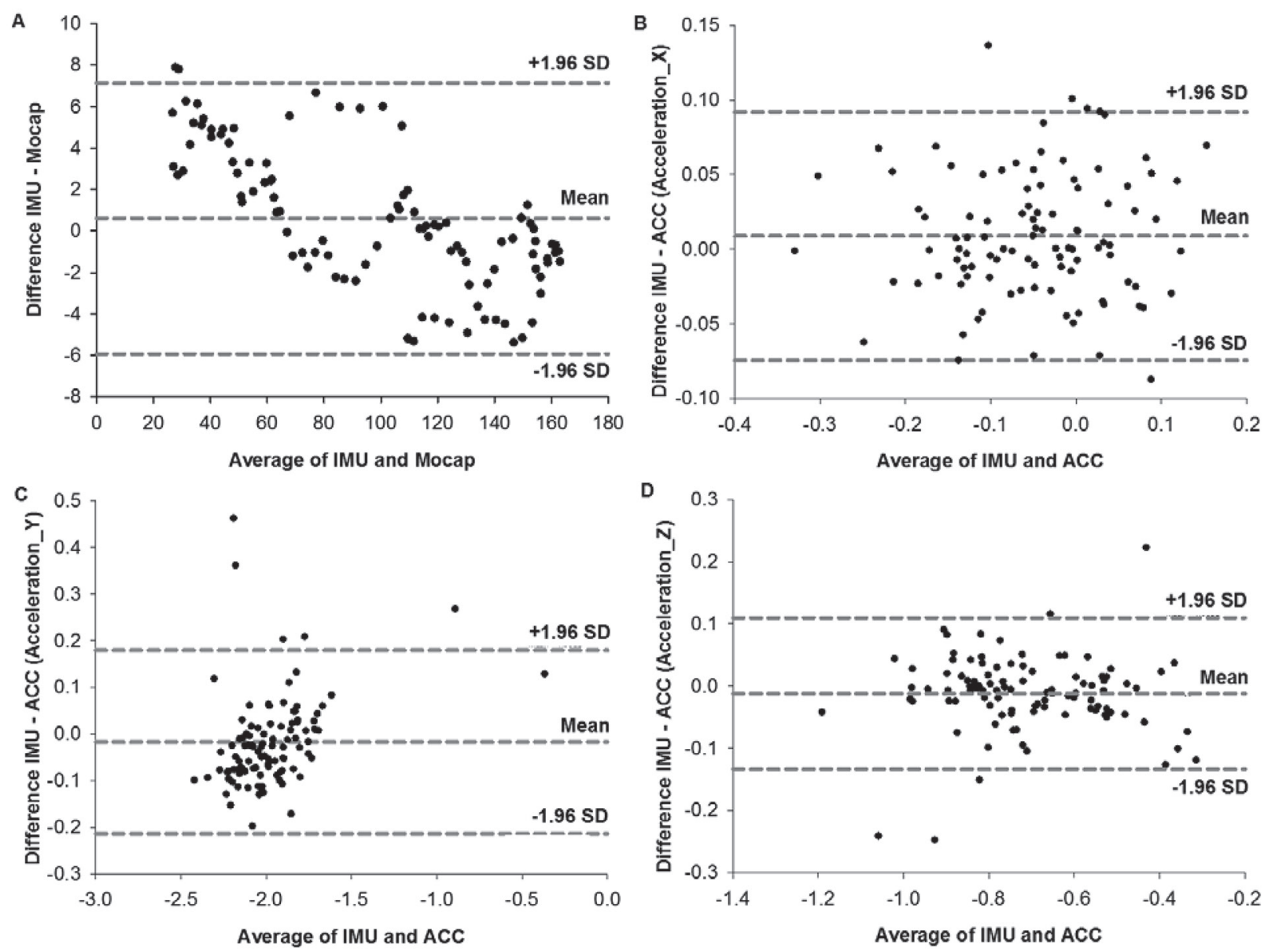

Figure 3. (A) Bland-Altman Plot representing the direction angle of the IMU comparisons to reference system (Mocap). (B-D) Bland-Altman Plot representing the 3-axes accelerations of the IMU comparison to reference system (ACC). Mean bias and error lines of $95 \%$ LOA are included. The bias between two measurements is uniform over the range of measurements.

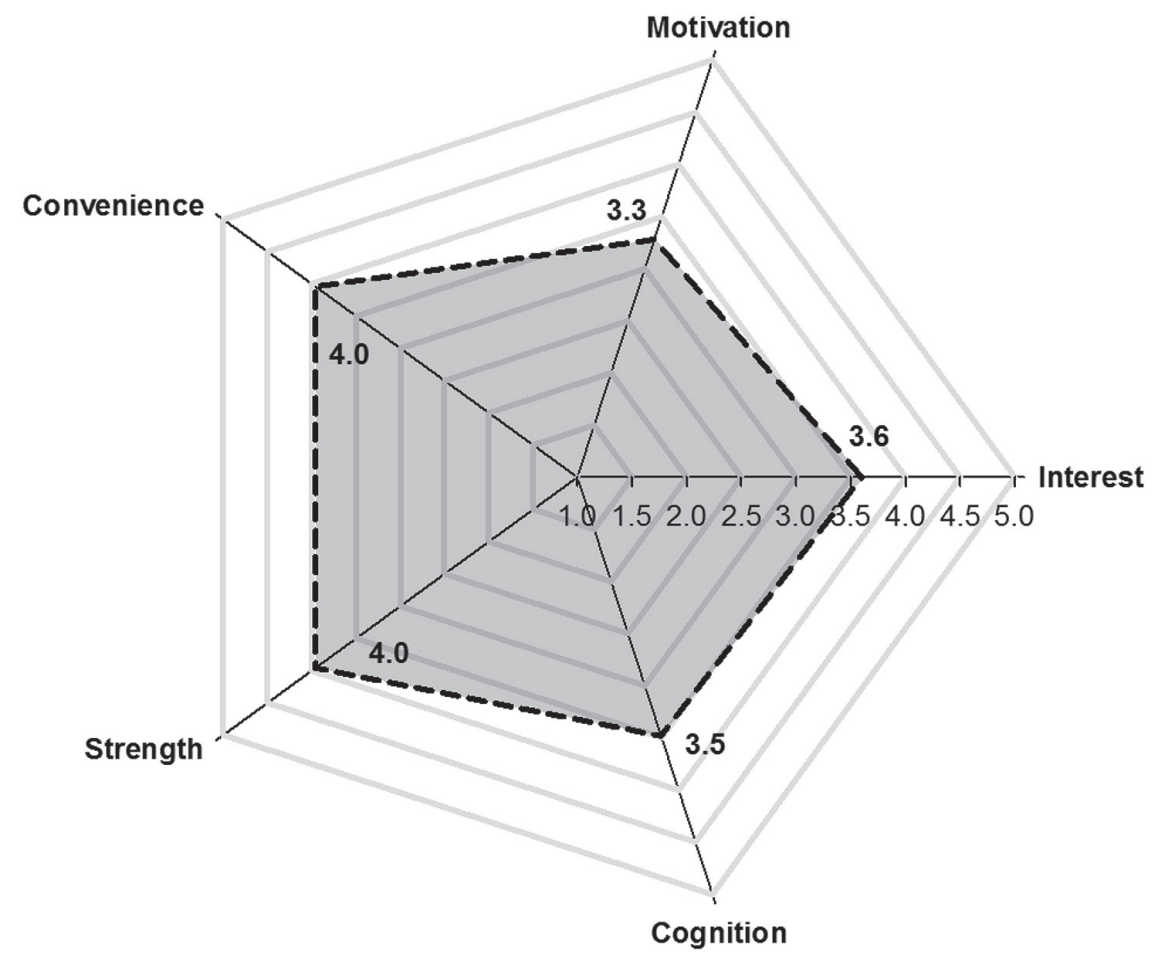

Figure 4. Usability results by the questionnaire. 


\section{Usability of the SEA}

Most participants positively answered to all questions about interest, motivation, convenience, strength and cognitive benefits ( $>66 \%$ ) of the SEA. A summary of usability results can be found in Figure 4.

\section{Discussion}

This study evaluated a new smart exercise application which would be included into an integrated exercise application in the future. The application was validated with a commercial accelerometer and a motion analysis system, and its usability was evaluated by a questionnaire. We found that: (1) the IMU showed a relatively high agreement with the reference systems in both direction angle and 3-axes accelerations; (2) SEA functioned well with assessing the lunge motion and short-term memory, and providing visual/auditory feedback; (3) most of the participants positively answered the questions related to interest, convenience to use, and effectiveness on physical/cognitive functions related to exercise with our application. These findings are discussed in detail next.

\section{Accuracy}

This application was programmed to calculate angular displacement of the body and determine how accurately the users face their body to the direction required during a lunge exercise. Thus, direction angle was one of the main kinematic variables to validate in this study. Our application showed a low RMSE value ( $<5$ degrees) and a high correlation coefficient $(r=.99)$ of angle displacement data, compared to the reference system. Low bias from Mocap measurement and uniform bias over the full range of motion shown in the BlandAltman plot also support these results. A previous study compared inertial measurement units with a motion capture system to validate their system. The results have shown that Pearson's correlation coefficients were between .80 and .94 and RMSE was in the range of 1.7 to 4.8 degrees during the lunge (Tang, et al., 2015). Another study evaluating the performance of the lunge exercise also indicated $83-90 \%$ accuracy using inertial measurement sensors (Whelan, et al., 2016). Compared to these previous studies, the accuracy of angle measurement of our application is similar or slightly higher.

The data from the accelerometer are the key variables to assess correct movements and to count the number of forward lunges. Our results were found to have high correlations and the lowest error sizes, especially y- and z-axes accelerations to assess correct movements. The accelerations during forward lunges were similar to those of Mocap and the ACC with minimal bias (accelerations: -0.02 to 0.01 and LOAs of -0.21 to $0.18 \mathrm{~m} / \mathrm{s}^{2}$ ).
Previous studies have reported that Pearson's correlation coefficients were between .75 and .99 of 3 -axes accelerations (Lee, Sutter, Askew, \& Burkett, 2010; Winter, Lee, Leadbetter, \& Gordon, 2016). Our results are equivalent to previous research that reported similar outcomes comparing 3-axes accelerations.

\section{Functionality}

All participants understood how to create sounds by performing the lunges within a 5 -minute practice. Based on the accurate kinematic data from the IMU sensor, our application created the sound as supposed (100\%, 164 of 164 trials) which could improve motivation (Rosati, et al., 2012). Several popular game systems such as Wii, Xbox, and PlayStation included similar functions (i.e., creating task-related audio/video feedback). However, all these systems require their own specific sensors and have limitations in mobility, and it is rare with a smart application with wearable sensors. In addition, providing feedback if the users memorized the order of four notes correctly would be helpful to improve motivation and short-term memory function (Kitazawa, et al., 2015; Teixeira, et al., 2013). Even though a 4-note task only was tested in this test, longer sequence (up to 8-note task) will be more challenging and would be useful in the longterm training study with a special population who want to improve their physical and cognitive function.

\section{Usability of the SEA}

As shown in the previous studies (Sale, Jacobs, MacDougall, \& Garner, 1990; Stone \& Coulter, 1994), our participants have positive perception about the effect of the lunge exercise. Ninety two percent of participants felt that the SEA could help strengthen their leg muscles. In addition, our SEA was developed to improve cognitive function as well, particularly short-term memory which is essential for functioning in daily life. Even though participants in the present study were asked to memorize a short sequence (four sounds) only, approximately $90 \%$ of participants believed that our SEA could improve their cognitive function, which is a positive sign for the success of our SEA. However, further research is needed to investigate cognitive functions after using the SEA for an intervention purpose because it was not directly measured in this study.

About $80 \%$ of participants answered that our application was easy to use. Even though only $24 \%$ of them had experience with SEA, they are all young adults and most of them are already familiar with smartphones. Future tests should include diverse populations including middle and older aged individuals who have relatively limited experience with 
smart devices. About $60 \%$ of participants answered that our application can cause interest to work out, which is a somewhat positive response. However, only $40 \%$ of participants responded that our application would improve motivation to exercise. We might need to improve functionality (or exercise enhancement) or add different functions to improve motivation to exercise, considering the answers from the questionnaire. Further study is warranted.

Overall, our SEA demonstrated accurate kinematic assessment of accelerations and directions, assessed the lunge motion correctly, and created the appropriate auditory feedback on the short-term memory task. In addition, the high rate of positive response suggested the success of the application in future use. However, the low positive response on motivation suggested that our application needs to be improved. It may be useful in long-term interventions such as home-based training aimed at increasing physical activity levels and cognitive functions with a healthy or a clinical population.

\section{References}

Alkjær, T., Henriksen, M., Dyhre-Poulsen, P., \& Simonsen, E.B. (2009). Forward lunge as a functional performance test in ACL deficient subjects: Test-retest reliability. The Knee, 16(3), 176-182.

Atkinson, R.C., \& Shiffrin, R.M. (1971). The control processes of short-term memory. Scientific American, 225(2), 82-91.

Brooke, J. (1996). SUS - A quick and dirty usability scale. In P.W. Jordan, B. Thomas, B. Weerdmeester \& I.L. McClelland (Eds.), Usability Evaluation in Industry (pp. 189 -194). London, Bristol: Taylor \& Francis.

Chen, C., Jafari, R., \& Kehtarnavaz, N. (2016). A real-time human action recognition system using depth and inertial sensor fusion. IEEE Sensors Journal, 16(3), 773-781.

Farrokhi, S., Pollard, C.D., Souza, R.B., Chen, Y.J., Reischl, S., \& Powers, C.M. (2008). Trunk position influences the kinematics, kinetics, and muscle activity of the lead lower extremity during the forward lunge exercise. Journal of Orthopaedic and Sports Physical Therapy, 38(7), 403-409. doi:10.2519/jospt.2008.2634

Fitzgerald, D., Foody, J., Kelly, D., Ward, T., Markham, C., McDonald, J., \& Caulfield, B. (2007). Development of a wearable motion capture suit and virtual reality biofeedback system for the instruction and analysis of sports rehabilitation exercises. In Proceedings of the 29th Annual International Conference of the IEEE and EMBS, Dublin, 2007.

Gowing, M., Ahmadi, A., Destelle, F., Monaghan, D.S., O’Connor, N.E., \& Moran, K. (2014). Kinect vs. low-cost inertial sensing for gesture recognition. In C. Gurrin, F. Hopfgartner, W. Hurst, H. Johansen, H. Lee \& N. O'Connor (Eds.), MultiMedia Modeling, Procedings of the $20^{\text {th }}$ Anniversary International Conference ( $\mathrm{pp}$. 484-495). Cham et al.: Springer.

Heijne, A., Fleming, B.C., Renstrom, P.A., Peura, G.D., Beynnon, B.D., \& Werner, S. (2004). Strain on the anterior cruciate ligament during closed kinetic chain exercises. Medicine and Science in Sports and Exercise, 36(6), 935-941.

Henriksen, M., Alkjær, T., Simonsen, E.B., \& Bliddal, H. (2009). Experimental muscle pain during a forward lunge The effects on knee joint dynamics and electromyographic activity. British Journal of Sports Medicine, 43(7), 503-507.

Institute of Electronic Music and Acoustics. (2016). Pure Data/on-line/. Retrieved April 6, 2017 from: https://puredata. info/.

Jönhagen, S., Halvorsen, K., \& Benoit, D.L. (2009). Muscle activation and length changes during two lunge exercises: Implications for rehabilitation. Scandinavian Journal of Medicine and Science in Sports, 19(4), 561-568.

Kalman, R.E. (1960). A new approach to linear filtering and prediction problems. Journal of Basic Engineering, 82(1), $35-45$.

Kitazawa, K., Showa, S., Hiraoka, A., Fushiki, Y., Sakauchi, H., \& Mori, M. (2015). Effect of a dual-task net-step exercise on cognitive and gait function in older adults. Journal of Geriatric Physical Therapy, 38(3), 133-140.

Leardini, A., Lullini, G., Giannini, S., Berti, L., Ortolani, M., \& Caravaggi, P. (2014). Validation of the angular measurements of a new inertial-measurement-unit based rehabilitation system: Comparison with state-of-the-art gait analysis. Journal of Neuroengineering and Rehabilitation, 11(1), 136.

Lee, J.B., Sutter, K.J., Askew, C.D., \& Burkett, B.J. (2010). Identifying symmetry in running gait using a single inertial sensor. Journal of Science and Medicine in Sport, 13(5), 559-563.

O’Reilly, M., Duffin, J., Ward, T., \& Caulfield, B. (2017). Mobile app to streamline the development of wearable sensorbased exercise biofeedback systems: System development and evaluation. JMIR Rehabilitation and Assistive Technologies, 4(2), e9.

O’Reilly, M.A., Whelan, D.F., Ward, T.E., Delahunt, E., \& Caulfield, B. (2017). Classification of lunge biomechanics with multiple and individual inertial measurement units. Sports Biomechanics, 16(3), 342-360. doi:10.1080/14 763141.2017.1314544 
Picerno, P. (2017). 25 years of lower limb joint kinematics by using inertial and magnetic sensors: A review of methodological approaches. Gait and Posture, 51, 239-246.

Rosati, G., Oscari, F., Spagnol, S., Avanzini, F., \& Masiero, S. (2012). Effect of task-related continuous auditory feedback during learning of tracking motion exercises. Journal of Neuroengineering and Rehabilitation, 9(1), 79.

Sale, D., Jacobs, I., MacDougall, J., \& Garner, S. (1990). Comparison of two regimens of concurrent strength and endurance training. Medicine and Science in Sports and Exercise, 22(3), 348-356.

Seizova-Cajic, T., \& Azzi, R. (2010). A visual distracter task during adaptation reduces the proprioceptive movement aftereffect. Experimental Brain Research, 203(1), 213-219.

Sourceforge. (2016). JKalman /on-line/. Retrieved April 6, 2017 from: https://sourceforge.net/projects/jkalman/.

Stone, W.J., \& Coulter, S.P. (1994). Strength/endurance effects from three resistance training protocols with women. Journal of Strength and Conditioning Research, 8(4), 231-234.

Tang, Z., Sekine, M., Tamura, T., Tanaka, N., Yoshida, M., \& Chen, W. (2015). Measurement and estimation of 3D orientation using magnetic and inertial sensors. Advanced Biomedical Engineering, 4, 135-143.

Teixeira, C.V.L., Gobbi, S., Pereira, J.R., Vital, T.M., Hernandéz, S.S.S., Shigematsu, R., \& Gobbi, L.T.B. (2013). Effects of square-stepping exercise on cognitive functions of older people. Psychogeriatrics, 13(3), 148-156.

Whelan, D., O’Reilly, M., Ward, T., Delahunt, E., \& Caulfield, B. (2016). Evaluating performance of the lunge exercise with multiple and individual inertial measurement units. In J. Favela \& N. Weibel (Eds.), Proceedings Proceedings of the 10th EAI International Conference on Pervasive Computing Technologies for Healthcare (pp. 102-108). Brussels: ICST.

Winter, S.C., Lee, J.B., Leadbetter, R.I., \& Gordon, S.J. (2015). Validation of a single inertial sensor for measuring running kinematics overground during a prolonged run. Journal of Fitness Research, 5(1), 14-23.

Submitted: March 30, 2018

Accepted: October 24, 2018

Published Online First: March 25, 2019

Correspondence to:

Assist. Prof. Tejin Yoon, Ph.D.

Department of Physical Education

Kangwon National University

1 Gangwondaehak-gil, Chuncheon, Republic of Korea

Phone: (82) 33-250-6787

E-mail: tyoon@kangwon. ac.kr 